\title{
Patient exposure in the basic science classroom enhances differential diagnosis formation and clinical decision-making
}

Justin G. Peacock, Joseph P. Grande

Purpose: The authors proposed that introducing real patients into a pathology classroom early in medical education would help integrate fundamental principles and disease pathology with clinical presentation and medical history.

Methods: Three patients with different pathologies described their history and presentation without revealing their diagnosis. Students were required to submit a differential diagnosis in writing, and then they were able to ask questions to arrive at the correct diagnosis. Students were surveyed on the efficacy of patient-based learning.

Results: Average student scores on the differential diagnosis assignments significantly improved $32 \%$ during the course. From the survey, $72 \%$ of students felt that patient encounters should be included in the pathology course next year. $74 \%$ felt that the differential diagnosis assignments helped them develop clinical decision-making skills. $73 \%$ felt that the experience helped them know what questions to ask patients. $86 \%$ felt that they obtained a better understanding of patients' social and emotional challenges.

Discussion: Having students work through the process of differential diagnosis formulation when encountering a real patient and their clinical presentation improved clinical decision-making skills and integrated fundamental concepts with disease pathology during a basic science pathology course. 
2 Patient exposure in the basic science classroom enhances differential diagnosis formation and 3 clinical decision-making.

4

5 Justin G. Peacock, M.D., Ph.D. ${ }^{1}$ and Joseph P. Grande, M.D., Ph.D. ${ }^{2}$

${ }^{1}$ Department of Graduate Medical Education

8 San Antonio Uniformed Services Health Education Consortium

9 San Antonio, TX, USA

$10{ }^{2}$ Department of Laboratory Medicine and Pathology

11 Mayo Clinic College of Medicine

12 Rochester, MN, USA

13

14 Running title

15 Patient exposure in pre-clinical course.

\section{Corresponding Author}

Justin G. Peacock, M.D., Ph.D.

19 Department of Graduate Medical Education

20 San Antonio Uniformed Services Health Education Consortium

213551 Roger Brook Dr.

22 Fort Sam Houston, TX 78234

23 USA

25 justin.g.peacock.mil@mail.mail

$26 \quad$ (507) 398-4304 


\section{Abstract}

\section{Purpose}

31 The authors proposed that introducing real patients into a pathology classroom early in medical

32 education would help integrate fundamental principles and disease pathology with clinical presentation

33 and medical history.

\section{Methods}

35 Three patients with different pathologies described their history and presentation without revealing

36 their diagnosis. Students were required to submit a differential diagnosis in writing, and then they

37 were able to ask questions to arrive at the correct diagnosis. Students were surveyed on the efficacy of 38 patient-based learning.

\section{Results}

40 Average student scores on the differential diagnosis assignments significantly improved $32 \%$ during

41 the course. From the survey, $72 \%$ of students felt that patient encounters should be included in the

42 pathology course next year. $74 \%$ felt that the differential diagnosis assignments helped them develop

43 clinical decision-making skills. $73 \%$ felt that the experience helped them know what questions to ask

44 patients. $86 \%$ felt that they obtained a better understanding of patients' social and emotional

45 challenges.

\section{Discussion}

47 Having students work through the process of differential diagnosis formulation when encountering a

48 real patient and their clinical presentation improved clinical decision-making skills and integrated

49 fundamental concepts with disease pathology during a basic science pathology course. 
52 A major challenge during medical school is making the leap from the basic science years to patient

53 encounters in the clinical setting. One difficulty involves forming differential diagnoses for a patient's

54 problem. Forming a differential diagnosis is critical to ordering proper clinical testing and

55 appropriately managing clinical disease. Unfortunately, most differential diagnosis education comes

56 during the third-year clerkships when the medical student is thrown into unfamiliar clinical situations

57 and responsibilities. Few attempts have been reported in the literature aimed at teaching differential

58 diagnosis and clinical decision-making in the early "basic science" years of medical education (Fulop,

59 1985; Duque, Gold \& Bergman, 2003; Gesundheit et al., 2009; Jacobson et al., 2010; Gunning \& Fors, 60 2012).

Some approaches in "early clinical exposure" have focused on the use of clinical cases and

62

63

64

65

66

67

68

standardized or virtual standardized patients to train medical students in clinical reasoning (Gesundheit et al., 2009; Jacobson et al., 2010; Gunning \& Fors, 2012). The use of clinical cases and standardized patients has been well studied and documented in the medical education literature (Tamblyn et al., 1991; TAMBLYN et al., 1991; Ainsworth, 1991; Colliver et al., 1998; Williams, 2004). While standardized patients have a long history in medical education, their use also has identified problems, including subjective biases in the standardized patients, inaccuracies, and unrealistic portrayals of patient experiences (Tamblyn et al., 1991; Williams, 2004).

At the Mayo Medical School, we have been teaching differential diagnosis in the context of the first-year Pathology course for a number of years. Students are taught how to form differential diagnoses and begin the initial steps of clinical decision-making (Martin et al., 2014). In the past, differential diagnosis was taught in the context of short patient cases that illustrated common human 
73 pathologies. We wanted to try a pilot study to bring actual patients with true pathologies into the basic

74 science classroom, early in undergraduate medical education, to see if students could delineate a

75 differential diagnosis for their pathologies. We wanted to help students integrate fundamental

76 principles of the basic sciences with a patient's clinical presentation, disease pathology, and course of

77 disease. We also wanted to increase students' awareness of the important aspects of clinical histories

78 and appropriate diagnostic testing or questioning in arriving at an accurate diagnosis. We hypothesized

79 that actual patients with clinical pathologies would provide even more important learning with regard

80 to clinical decision-making for the students.

81

82

83

84

85

\section{Materials and Methods}

This study was submitted to the Mayo Clinic Institutional Review Board for approval. It was determined in verbal communications not to require IRB approval, because it was conducted in the context of required assignments in an educational course.

In this pilot study, 47 first-year pathology students were introduced to three different volunteer patients with actual disease pathologies to present their clinical histories. The patients had previously expressed an interest in sharing their clinical history with medical students to faculty and staff at the clinic. The three patients came into the classroom on three different occasions during the course and spent approximately 30 minutes discussing the history and clinical scenario leading up to their diagnosis, without revealing their diagnosis. Immediately after the patient finished their history, students then completed an online assignment, which asked for their top three diagnoses (beginning with the most likely), an explanation of the aspects of the case that led to their most likely diagnosis, and additional questions or information that would help to confirm their differential (Figure 1). The 
95 goal with the questions was not for the students to obtain the exact, true diagnosis, but for them to use

96 their clinical reasoning skills to narrow down a reasonable differential diagnosis list based on the

97 patient's history. We wanted the students to justify the rationale for their differential list and to

98 formulate additional questions or tests that they would like to use to narrow down the differential list.

99 Following submission of the online assignment, students were then permitted to ask the patients

100 additional questions to determine the true diagnosis as a class. They were also given time to ask

101 questions regarding the social, behavioral, economic, and other impacts of the disease on the patients'

102 lives.

103

104

105

106

107

108

109

110

111

112

As the patient cases were not straightforward, simple, first-year cases, it was stressed to both the students and the teaching assistants that the goal of the assignments was not to obtain the exact, correct diagnosis, but to formulate a reasonable differential diagnosis list based on the patient's history. We specifically sought out patients with more complicated or multi-system diagnoses to expand the differential diagnoses that students could assemble for a given patient history. Consequently, the cases and the grading rubric allowed for a wider variability in the differential diagnoses compared with simpler medical case presentations. Teaching assistants utilized an established grading rubric and grading methodology used in other differential diagnosis assignments in the course to grade the assignments on an 18-point scale (Figure 1)(Martin et al., 2014). Student scores on the assignments were compared using ANOVA with Tukey-Kramer post-processing at the $0.05 \alpha$ level.

At the conclusion of the course a survey was conducted among the students to determine the impact of patient encounters on their understanding of pathology, differential diagnosis formation, clinical reasoning, and patient empathy. The survey results were tallied on the basis of a five-point Likert scale. For simplicity in the summary table, strong and very strong agreement ratings were 
117 grouped into the agreement column and strong and very strong disagreement ratings were grouped into

118 the disagreement column.

120 Results

121 We piloted bringing volunteer patients with actual disease into a first-year pathology course to help 122 students develop their differential diagnosis, history-taking, and basic clinical decision-making skills.

123 The students' previous first-year courses included genetics, anatomy, and histology. The first volunteer

124 patient presented his history of liver cirrhosis secondary to alpha- 1 antitrypsin deficiency during the

125 first week of the course. The differential assignments were graded by teaching assistants using the

126 grading rubric shown in Figure 1 and using a previously described grading methodology (Martin et al.,

127 2014). The first assignment resulted in an average score of $8.8 \pm 3.1$ out of 18 (Figure 2). During the

128 pathology course, lectures, assignments, and teaching assistant feedback was devoted to helping

129 students learn how to develop differential diagnoses, including the VITAMIN CDE methodology

130 (Martin et al., 2014), how to ask appropriate questions of patients, and how to make basic diagnostic

131 decisions.

The second volunteer patient was a pediatric patient who experienced a biliary leak and

133 infection secondary to liver transplantation. The mother and patient presented the patient's history to

134 the class approximately 3 weeks after the first patient. The second assignment resulted in an average

135 score of $9.7 \pm 2.7$ (Figure 2), which was higher than the first assignment, but not significantly higher.

136 Due to scheduling conflicts, the third patient presented his case of heart failure secondary to

137 hypertrophic obstructive cardiomyopathy approximately one week after the second patient. The

138 average score on this third assignment was $11.6 \pm 2.7$, which was significantly higher than both the 
139 first and second assignments, $\mathrm{p}<0.0001$ and $\mathrm{p}=0.0043$, respectively (Figure 2 ). We found that the

140 students' grades on the patient encounter differential diagnosis assignments improved significantly by

$14132 \%$ over the course of the block (Figure 2).

At the end of the pathology block, students were asked to fill out a survey regarding their

143 experiences with the patient encounter differential diagnosis assignments (Table 1). Overall, the

144 students felt that the patient encounter experiences should be a continuing part of the pathology block

145 and other first-year medical school courses ( $72.3 \%$ and $66.0 \%$, respectively). Importantly, students

146 strongly felt that the patient encounter experiences helped them develop clinical decision-making

147 skills, know what questions to ask patients, and understand social and emotional challenges that

148 patients face during disease $(74.5 \%, 74.5 \%$, and $87.2 \%$, respectively). Interestingly, the students still

149 indicated that they did not feel more comfortable facing patient encounters in the clinic after these

150 experiences (27.7\%). Students also indicated that they would have liked to have had patients with

151 simpler, more common pathologies than those presented (data not shown).

\section{Discussion}

154 In this study, we have demonstrated that real patient encounters in the basic science classroom coupled

155 with assignments aimed at clinical decision-making may improve clinical skills and help to provide

156 clinical context to the basic sciences they are learning. We have shown that actual, volunteer patients

157 can be brought into a first-year, basic science classroom to serve as patient educators, helping students

158 learn to ask the right questions, formulate differential diagnoses, and understand the nonmedical

159 challenges that patients endure. We believe that this coupling of the basic and clinical sciences in the 
160 early years of medical school is important to help students more readily and confidently transition from

161 the classroom to the clinical setting.

162

163

164

165

166

167

168

169

170

171

172

173

174

175

176

177

178

179

180

181

Other institutions have provided beginning medical students with early clinical exposure, through the use of clinical case scenarios and simulated patients (Fulop, 1985; Duque, Gold \& Bergman, 2003; Gesundheit et al., 2009; Jacobson et al., 2010; Gunning \& Fors, 2012). The challenge with these simulated experiences lies in their very nature, namely, they are simulated or artificial experiences (Tamblyn et al., 1991; Williams, 2004). Actual patient encounters provide realistic exposure to clinical scenarios. They can provide clinical context and psychosocial factors that cannot be considered in typical standardized patient scenarios. We did not focus on the students coming up with the exact, correct diagnosis at this early stage, because we felt that the process involved in formulating and justifying a reasonable differential diagnosis list is more important in early medical education. In particular, we wanted students to broaden their differential in the context of the pathology that they were learning about in the course.

Another benefit of real patient exposure in the early medical school years lies in the development of empathy or emotional IQ. While not directly assessed in this study, an overwhelming percentage of students did report a greater appreciation for the social and emotional challenges that patients endure. It is not clear that the same increase in empathy could be obtained from simulated patient experiences (Colliver et al., 1998). Empathy or emotional IQ is an important attribute of professional physicians, particularly, with many studies showing a decline in empathy over the course of medical education (Neumann et al., 2011).

We understand that our study is limited in that it was a pilot study for bringing actual patients into the basic science classroom. We only were able to recruit the three patient volunteers during the 
182 first round of recruiting. While we felt that each patient scenario was appropriate for allowing students

183 to create a reasonable differential diagnosis list, we were not able to choose from among a wide variety

184 of patient scenarios. We also encountered some of the challenges in dealing with actual patients rather

185 than simulated patients, in that we were not able to decide when students would encounter patient

186 scenarios due to patient schedules. We also did not have fine control over how much or how little the

187 patients would discuss about their cases. The patients were instructed to give as much pertinent history

188 as they could without revealing the actual diagnoses being assessed. In the future, we hope to have a

189 larger pool of volunteer patients from which to decide the most appropriate patient encounters at

190 regular intervals during the course.

Interestingly, although students felt that the patient encounters increased their clinical decision-

192 making skills and helped them to know what types of questions to ask patients, many students still did

193 not feel an increase in confidence in dealing with patients (Table 1). Understanding that these students

194 are still first-year students, it would be interesting to know what knowledge, skills, and experiences

195 students require to feel confident going into clinical patient scenarios. It would also be interesting to

196 see how the patient educators would rate students with regards to empathy or emotional IQ during

197 these sessions to work on improving this professional characteristic. Unfortunately, in this study, we

198 did not collect a grade breakdown for the different grading rubric criteria from the teaching assistants,

199 only a total grade for the assignment. In the future, we would like to understand what specific areas

200 showed the most improvement during the course, i.e. differential formation, differential justification, or

201 clinical decision-making. Lastly, we would like to develop objective means to determine if the skills

202 taught in this course are being implemented in the clinical setting. 


\section{Conclusions}

205 Introducing actual patients into a first-year, basic science pathology classroom helped students to

206 develop differential diagnosis formation, history-taking, and basic clinical decision-making skills at an

207 early stage of undergraduate medical education. Students also reported that they were better able to 208 understand and appreciate the challenges that patients face during the course of their disease. 


\section{Disclaimer}

211 The view(s) expressed herein are those of the author(s) and do not reflect the official policy or position 212 of Brooke Army Medical Center, the U.S. Army Medical Department, the U.S. Army Office of the 213 Surgeon General, the Department of the Army, the Department of the Air Force and Department of 214 Defense or the U.S. Government.

215

\section{Declaration of Interest}

217 The authors do not have any conflicts of interest.

218 


\section{References}

221 Ainsworth MA. 1991. Standardized Patient Encounters. JAMA 266:1390.

222

Colliver JA, Willis MS, Robbs RS, Cohen DS, Swartz MH. 1998. Assessment of Empathy in a Standardized-Patient Examination. Teaching and Learning in Medicine 10:8-11.

Duque G, Gold S, Bergman H. 2003. Early Clinical Exposure to Geriatric Medicine in Second-Year Medical School Students--The McGill Experience. Journal of the American Geriatrics Society 51:544-548.

Fulop M. 1985. Teaching differential diagnosis to beginning clinical students. The American Journal of Medicine 79:745-749.

Gesundheit N, Brutlag P, Youngblood P, Gunning WT, Zary N, Fors U. 2009. The use of virtual patients to assess the clinical skills and reasoning of medical students: initial insights on student acceptance. Medical teacher 31:739-42.

Gunning WT, Fors UGH. 2012. Virtual patients for assessment of medical student ability to integrate clinical and laboratory data to develop differential diagnoses: comparison of results of exams with/without time constraints. Medical teacher 34:e222-8.

Jacobson K, Fisher DL, Hoffman K, Tsoulas KD. 2010. Integrated Cases Section: a course designed to promote clinical reasoning in year 2 medical students. Teaching and learning in medicine 22:3126.

Martin BA, Peacock JG, Warner LL, Wentworth AB, Adefusika JA, Bachman MJ, Drozdowicz LB, Suresh R, Grande JP. 2014. A Novel Metric and Feedback Template Improves Differential Diagnosis Formation Capabilities in Pre-Clinical Medical Students. Medical Science Educator 24:189-194.

Neumann M, Edelhäuser F, Tauschel D, Fischer MR, Wirtz M, Woopen C, Haramati A, Scheffer C. 2011. Empathy Decline and Its Reasons: A Systematic Review of Studies With Medical Students and Residents. Academic medicine : journal of the Association of American Medical Colleges:996-1009.

Tamblyn RM, Klass DJ, Schnabl GK, Kopelow ML. 1991. Sources of unreliability and bias in standardized-patient rating. Teaching and Learning in Medicine 3:74-85.

Tamblyn RM, Klass DJ, Schnabl GK, Kopelow ML. 1991. The accuracy of standardized patient presentation. Medical Education 25:100-109. 
250 Williams RG. 2004. Have standardized patient examinations stood the test of time and experience? 251 Teaching and learning in medicine 16:215-22.

252 


\section{Figures}

256 Figure 1. Questions and grading rubric for patient encounters.

257 The patient encounter questions given to the students are seen in black type, while the grading rubric is 258 seen in red type.

260 Figure 2. Box plot of student scores for patient encounter assignments.

261 Box plot with 25\% quartiles and median for the grades of students during the first, second, and third 262 patient encounters. One-factor ANOVA with repeated measures indicated a significant difference in 263 the grades between the $3^{\text {rd }}$ patient scores compared with the $1^{\text {st }}$ and $2^{\text {nd }}$ patient $\operatorname{scores}[\mathrm{F}$ Ratio $=$ $26412.1244, \mathrm{P}<0.0001]$. ${ }^{*} \mathrm{P}<0.05$, by post hoc Tukey-Kramer HSD. Significant pairings are designated 265 by a bracket connecting the pairings with an * above the bracket. 


\section{Tables}

272 Table 1. Survey summary for student survey regarding patient encounter experiences.

273 Survey statistics are listed as percentages of the total class responses $(N=47)$. Likert scores of

274 strong/very strong agree (4/5) are grouped together in agreement column, Likert scores of strong/very

275 strong disagree (1/2) are grouped together in disagreement column, and the rest (Likert score 3 ) are in 276 the neutral column.

277

278

279

280 
Figure 1

Figure 1. Questions and grading rubric for patient encounters.

The patient encounter questions given to the students are seen in black type, while the grading rubric is seen in red type.

Patient Encounter Grading Rubric (18 points possible)

Given the clinical presentation you have just heard:

1) What are your top three diagnoses, in order, beginning with the most likely? ( 8 pts. possible)

1. 0 -2 points ( 2 = excellent likelihood, 1 = good/likely, $0=$ unlikely/blank)

2. 0 -2 points ( 2 = excellent likelihood, 1 = good/likely, $0=$ unlikely/blank)

3. 0 -2 points ( 2 = excellent likelihood, $1=$ good/likely, $0=$ unlikely/blank)

For \#1, most likely diagnosis, if it truly is the most likely diagnosis out of the three possible diagnoses give 1 point.

If \#1 is the correct diagnosis give 1 more point

2) What aspects of the case lead you to your most likely diagnosis? (1-2 sentences) (4 points possible)

For each aspect of the history that corroborates with their most likely (\#1 above) diagnosis, give 1 point. up to a maximum of 4 points. For each incorrect aspect cited, subtract 1 point.

3) What other questions/information do you believe would help you confirm/justify your differential? (3-4 questions) (6 points possible)

0-2 points ( 2 = excellent question/info to confirm diagnosis, 1 = good ques/info, 0 = poor/blank ques/info)

0-2 points ( 2 = excellent question/info to confirm diagnosis, 1 = good ques/info, 0 = poor/blank ques/info)

0-2 points ( 2 = excellent question/info to confirm diagnosis, 1 = good ques/info, 0 = poor/blank ques/info) 


\section{2}

Figure 2

Figure 2. Box plot of student scores for patient encounter assignments.

Box plot with $25 \%$ quartiles and median for the grades of students during the first, second, and third patient encounters. One-factor ANOVA with repeated measures indicated a significant difference in the grades between the 3rd patient scores compared with the 1st and 2 nd patient scores [F Ratio $=12.1244, \mathrm{P}<0.0001]$. $* \mathrm{P}<0.05$, by post hoc Tukey-Kramer HSD. Significant pairings are designated by a bracket connecting the pairings with an * above the bracket. 


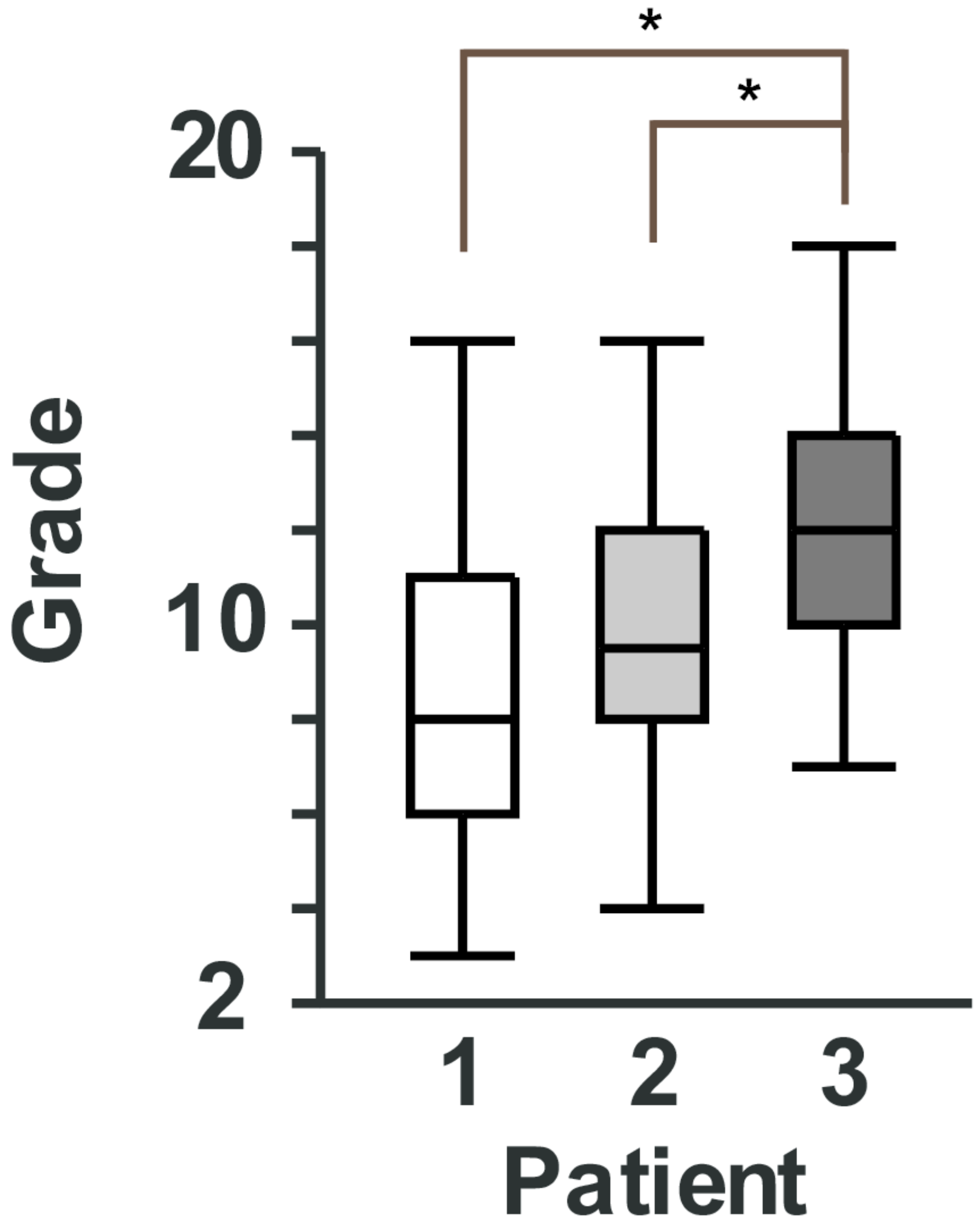


Table $\mathbf{1}$ (on next page)

Table 1

Table 1. Survey summary for student survey regarding patient encounter experiences.

Survey statistics are listed as percentages of the total class responses $(N=47)$. Likert scores of strong/very strong agree (4/5) are grouped together in agreement column, Likert scores of strong/very strong disagree (1/2) are grouped together in disagreement column, and the rest (Likert score 3) are in the neutral column. 


\section{Survey Statement}

Patient encounters should be incorporated into the Pathology block next year.

More patient encounters should be included

in the first-year courses.

The DDX assignments associated with the patient encounters help me develop differential diagnosis formation skills.

The DDX assignments associated with the patient encounters helped me develop clinical decision-making skills.

I have a better understanding of pathology through the patient encounter experiences.

The patient encounters helped me to better link a patient's pathology with the patient's clinical presentation.

The patient encounters helped me to better recognize key elements of clinical history and exam in a patient.

The patient encounters improved my confidence to interact with patients.

The patient encounters gave me a better sense of what questions would be important to ask patients.

The patient encounters gave me a better sense of what diagnostic procedures or test to order for patients.

The patient encounters gave me a better appreciation of the social and emotional challenges that patients go through.

The patient encounters helped to give you insight into what your clinical experience might be like.
Agreement

Neutral

Disagreement

72.3

12.8

14.9

66.0

17.0

17.0

57.4

17.0

23.4

74.5

10.6

14.9

53.2

21.3

25.5

68.1

14.9

14.9

66.0

23.4

10.6

27.7

31.9

40.4

74.5

12.8

12.8

63.8

6.4

29.8

87.2

8.5

4.3

68.1

19.1

10.6 